\title{
A NOTE ON COMPLEMENTATION IN LATTICES
}

L. R. WILCOX

The results of this note concern the notions of complementation and modularity in lattices. In the theories of modular lattices, for example, the theory of continuous geometries of von Neumann, the assumption of complementation has proved extremely powerful; the author has found that some analogue of this assumption is usually necessary in the study of non-modular lattices. ${ }^{1}$ If $L$ is a modular lattice with 0,1 , and if $a \in L$, then $x$ is a complement of $a$ in case $a+x=1, a x=0$. If $L$ has the property

$$
\text { each } a \in L \text { has a complement, }
$$

then it may be proved that

$$
a, b \in L \text { implies the existence of } b_{1} \leqq b \text { such that }
$$

$$
a+b_{1}=a+b, \quad a b_{1}=0 .
$$

Property (2) is the really useful one. Now if $L$ is any lattice with 0,1 , then (1) does not imply (2); moreover, even (2) seems too weak for most purposes. However, if in (2) we assume in addition that $\left(a, b_{1}\right)$ (or $\left(b_{1}, a\right)$ ) is a modular pair, then the usefulness of this assumption in analyzing the structure of $L$ is considerably increased. At the same time, of course, limitations are placed on $L$, and it is these which we study here.

Let $L$ be a lattice with 0 and 1 . If $b, c \in L$, we say that $(b, c)$ is a modular pair and write $(b, c) M$ if $(a+b) c=a+b c$ for $a \leqq c$. We write $(b, c) \perp$ if $b c=0$ and $(b, c) M$. The negation of $M$ is $M^{\prime}$. If $b c=0$ and $(b, c) M^{\prime}$ we write $b \| c$. If $\perp$ is a symmetric relation, $L$ is said to be $\perp$-symmetric; if $M$ is symmetric, $L$ is said to be $M$-symmetric. ${ }^{2}$

Definition 1. Let $a, b, b_{1} \in L$. Then $b_{1}$ is a right complement within $b$ of $a$ in $a+b$ if

$$
b_{1} \leqq b, \quad a+b_{1}=a+b, \quad\left(a, b_{1}\right) \perp .
$$

We say that $b_{1}$ is a left complement within $b$ of $a$ in $a+b$ if

$$
b_{1} \leqq b, \quad a+b_{1}=a+b, \quad\left(b_{1}, a\right) \perp .
$$

If $a \leqq b$, the phrase "within $b$ " is omitted; if $a+b=1$, the phrase

Presented to the Society, September 5, 1941; received by the editors September 24, 1941.

1 This Bulletin, abstract 47-5-208

${ }^{2}$ See L. R. Wilcox, Modularity in the theory of lattices, Annals of Mathematics, (2), vol. 40 (1939), pp. 490-505, for the elementary properties of the relations $M$ and $\perp$. 
"in $a+b$ " is omitted. $L$ is right (left) complemented if $a, b \in L$ implies the existence of $b_{1}$ such that $b_{1}$ is a right (left) complement within $b$ of $a$ in $a+b$. If $L$ is $\perp$-symmetric, right and left complements coincide, and the words "right" and "left" will be omitted.

THEOREM 1. Let $(a, b) M$. Then $b_{1}$ is a right complement within $b$ of $a$ in $a+b$ if and only if $b_{1}$ is a right complement of $a b$ in $b$. The forward implication holds also for left complements.

Proof. Suppose $b_{1} \leqq b, b_{1}+a=b+a,\left(a, b_{1}\right) \perp\left(\right.$ or $\left.\left(b_{1}, a\right) \perp\right)$. Then

$$
b_{1}+a b=\left(b_{1}+a\right) b=(b+a) b=b ;
$$

$\left(a b, b_{1}\right) \perp\left(\right.$ or $\left.\left(b_{1}, a b\right) \perp\right)$ is immediate. Suppose that $a b+b_{1}=b$, $\left(a b, b_{1}\right) \perp$. Then $b_{1} \leqq b$; moreover, $a+b_{1}=a+a b+b_{1}=a+b$. Clearly $a b_{1}=a b b_{1}=0$. Let $d \leqq b_{1}$, whence

$$
\begin{aligned}
(d+a) b_{1} & =(d+a) b b_{1}=(d+a b) b_{1} \\
& =d+a b b_{1}=d+a b_{1},
\end{aligned}
$$

and $\left(a, b_{1}\right) M$. Hence $\left(a, b_{1}\right) \perp$, and $b_{1}$ is a right complement within $b$ of $a$ in $a+b$.

We turn now to consequences of the assumption that $L$ is left complemented. The fundamental result follows.

THEOREM 2. If $L$ is left complemented, then $L$ is right complemented, the notions of right and left complement coincide, and $L$ is $M$-symmetric.

Proof. Let $(b, c) M$. There exists a left complement $c_{1}$ within $c$ of $b$ in $b+c$. Now let $b_{1} \leqq b$. Then by the second part of Theorem $1 c_{1}$ is a left complement of $b c$ in $c$, and

$$
\left(b_{1}+c\right) b=\left(b_{1}+b c+c_{1}\right) b=b_{1}+b c,
$$

whence $(c, b) M$. The remainder of the theorem is immediate.

Corollary. If $L$ is $\perp$-symmetric and complemented, then $L$ is $M$ symmetric.

REMARK. It is false that if $L$ is right complemented then $L$ is left complemented. For, in the right complemented lattice consisting of 0,1 , three points $p, q, r$, and a line $s$ with $s>p, q$ but $s \ngtr r$, the element $s$ has no left complement (in 1).

We shall henceforth assume that $L$ is left complemented. It is now possible to prove a converse of Theorem 1 .

THEOREM 3. Let $a, b \in L$. If every complement $b_{1}$ within $b$ of $a$ in $a+b$ is a complement of $a b$ in $b$, then $(a, b) M$. 
Proof. Let $d \leqq b$, and let $d_{1}$ be a complement within $d$ of $a$ in $a+d$. Then

$$
d_{1}+a=d+a, \quad\left(d_{1}, a\right) \perp .
$$

Now let $b^{\prime}$ be a complement within $b$ of $d+a$ in $b+a$. Then

$$
b^{\prime}+d_{1}+a=b^{\prime}+d+a=b+a ;
$$

moreover, $\left(b^{\prime}, d+a\right) \perp$, whence $\left(b^{\prime}, d_{1}, a\right) \perp$, and $\left(b^{\prime}+d_{1}, a\right) \perp$. Define $b_{1} \equiv b^{\prime}+d_{1}$. Then $b_{1} \leqq b, b_{1}+a=b+a,\left(b_{1}, a\right) \perp$, that is, $b_{1}$ is a complement within $b$ of $a$ in $a+b$. By the hypothesis, $b_{1}$ is a complement of $a b$ in $b$, whence $b_{1}+a b=b$. Now

$$
\begin{aligned}
(d+a) b & =(d+a)\left(b_{1}+a b\right) \\
& =\left(\left(a b+d_{1}\right)+b^{\prime}\right)(d+a) \\
& =a b+d_{1} \leqq d+a b
\end{aligned}
$$

since $a b+d_{1} \leqq d+a,\left(b^{\prime}, d+a\right) \perp$. But $d+a b \leqq(d+a) b$, whence $(d+a) b$ $=d+a b$, and $(a, b) M$.

CoRollaRy 1. $(a, b) M$ if and only if every complement within $b$ of $a$ in $a+b$ is a complement of $a b$ in $b$.

Corollary 2. $(a, b) M^{\prime}$ if and only if there exists a complement $b_{1}$ within $b$ of $a$ in $a+b$ such that $b_{1}+a b<b$.

Corollary 3. $a \| b$ if and only if $a b=0$ and there exists $b_{1}<b$ such that $b_{1}+a=b+a$ and $\left(b_{1}, a\right) \perp$.

REMARK. It should be observed that Corollary 3 justifies our use of the symbol $\|$. Let, for example, $L$ be a euclidean (or affine) geometry. Parallel spaces in $L$ are then characterized by the condition $a b=0$ and the existence of a space $b_{1}<b$ such that $b_{1}+a=b+a$; if $b_{1}$ is chosen as a minimal space with these properties, and it can be so chosen, then $\left(b_{1}, a\right) \perp$.

It is easily seen ${ }^{3}$ that an affine geometry is a left complemented lattice. Indeed, it may be shown that the property

$$
b c \neq 0 \text { implies }(b, c) M
$$

holds in an affine geometry. Property (3) may be stated also in the following way. Define the set $M_{L}^{\prime}$ as the set $\left[b c ;(b, c) M^{\prime}\right]$. Then

$$
M_{L}^{\prime} \subset[0] \text {. }
$$

It is of interest to investigate the nature of the set $M_{L}^{\prime}$ for our general

${ }^{3}$ L. R. Wilcox, loc. cit., pp. 496-499. 
left complemented lattice $L$. It might appear that $M_{L}^{\prime}$ is an ideal; that this is false will be shown by an example. However, the next theorem shows that $M_{L}^{\prime}$ has one of the properties of an ideal.

Lemma 1. Let $(b, c) M^{\prime}$ and $a<b c$. Then there exists $b^{\prime}<b$ with $a=b^{\prime} c$ and $\left(b^{\prime}, c\right) M^{\prime}$. Moreover, there exists $x \leqq b$ with $x \| c$.

Proof. Let $x$ be a complement of $b c$ in $b$, whence $x+b c=b,(x, b c) \perp$. Define $b^{\prime} \equiv a+x \leqq b$. Then

$$
b^{\prime} c=(a+x) c=(a+x) b c=a .
$$

Suppose that $\left(b^{\prime}, c\right) M$, that is, $(a+x, c) M$. Then $c_{1} \leqq c$ implies

$$
\begin{aligned}
\left(c_{1}+b\right) c & =\left(c_{1}+x+b c\right) c \\
& =\left(c_{1}+x+a+b c\right) c \\
& =c_{1}+b c+(x+a) c \\
& =c_{1}+b c,
\end{aligned}
$$

contrary to $(b, c) M^{\prime}$. Hence $\left(b^{\prime}, c\right) M^{\prime}$. The second part follows by putting $a=0$.

Theorem 4. If $x \in M_{L}^{\prime}$ and $y \leqq x$, then $y \in M_{L}^{\prime}$.

Proof. Immediate from Lemma 1.

We turn now to a general example of a left complemented lattice. Let $\Lambda$ be a complemented modular lattice with operations $\oplus, \otimes$, and let $\infty$ be any element of $\Lambda$; let $L$ be a subset of $\Lambda$ with the following properties:

$$
\begin{aligned}
& \mathfrak{a} \equiv[x ; x \leqq \infty] \subset L ; 1 \in L ; \\
& x \in L, x \in \mathfrak{a}, y \geqq x \text { implies } y \in L ; \\
& x \in L, y \leqq x \text { implies the existence of } z \in L \text { such that } \\
& \quad z \oplus y=x, \quad z \otimes y=0 .
\end{aligned}
$$

A special case is the following. $\Lambda$ is a projective geometry, and $\infty$, $h \in \Lambda, 0<\infty<h<1, L^{\prime} \equiv[x ; x \leqq h, x$ not $\leqq \infty], L \equiv \Lambda-L^{\prime}$. Then $L$ obviously satisfies (4) and (5), and (6) holds by virtue of the irreducibility of $\Lambda$. If $h$ is a hyperplane, that is, $h$ is a maximal element less than 1 , then $L$ is an affine geometry (for example, $\Lambda$ with $h$ as ideal hyperplane) with the ideal elements of $\mathfrak{a}$ restored.

Theorem 5. $L$ is a lattice in which the operations + , a are given by

$$
a+b=a \oplus b, \quad a b= \begin{cases}a \otimes b \quad \text { if } \quad a \otimes b \in L \\ a \otimes b \otimes \infty \text { otherwise. }\end{cases}
$$


Proof. To show that $a \oplus b$ is a least upper bound of $a, b$ in $L$ it suffices to prove that $a, b \in L$ implies $a \oplus b \in L$. If both $a, b \in \mathfrak{a}$, then $a \oplus b \in L$ by (4). Hence suppose $a \oplus \mathfrak{a}$; by (5) $a \oplus b \in L$. We shall prove directly that $a b$ as defined in (7) is effective as greatest lower bound of $a, b$ in $L$. Clearly $a b \leqq a, b$; by (4) $a b \in L$. Let $c \in L, c \leqq a, b$. Then $c \leqq a \otimes b$. If $c \notin \mathfrak{a}$, then $a \otimes b \in L$, and $c \leqq a b$ by (7). If $c \in \mathfrak{a}$, then $c \leqq a \otimes b \otimes \infty \leqq a b$.

Corollary. If $a \in \mathfrak{a}, b \in L$, then $a b=a \otimes b$.

Lemma 2. If $b, c \in L$, then $(b, c) M$ if and only if $b \otimes c \in L$.

Proof. First let $(b, c) M$, and suppose $b \otimes c \notin L$. Let $a \in L$ be a complement of $b \otimes c$ in $c$, existent by (6). Then

$$
(a \oplus b) \otimes c=a \oplus(b \otimes c)=c,
$$

whence $(a \oplus b) \otimes c \in L$; thus by $(7)$

$a \oplus(b \otimes c)=(a \oplus b) \otimes c=(a+b) c=a+b c=a \oplus(b \otimes c \otimes \infty)$,

and it follows from the theory of modular lattices that $(b \otimes c) \otimes \infty$ $=b \otimes c$. By (4) this contradicts the hypothesis, whence $b \otimes c \in L$. Conversely, let $b \otimes c \in L$, and let $a \leqq c, a \in L$. If $a \notin \mathfrak{a}$, then $(a \oplus b) \otimes c \in L$, and by (7) and the modular law in $\Lambda$ we have

$$
(a+b) c=(a \oplus b) \otimes c=a \oplus(b \otimes c)=a+b c .
$$

Suppose $a \in \mathfrak{a}$. If $(a \oplus b) \otimes c \in L$, equalities (8) hold. If $(a \oplus b) \otimes c \notin L$, then

$$
\begin{aligned}
(a+b) c & =(a \oplus b) \otimes c \otimes \infty=(a \oplus(b \otimes c)) \otimes \infty \\
& =a \oplus(b \otimes c \otimes \infty) \leqq a+b c \leqq(a+b) c .
\end{aligned}
$$

By (8) and (9) we have $(b, c) M$.

Corollary. $L$ is $M$-symmetric.

Theorem 6. The lattice $L$ is left complemented.

Proof. Let $a, b \in L$, and let $b_{1} \in L$ be a complement of $a \otimes b$ in $b$, existent by (6). Then

$$
\begin{aligned}
& a+b_{1}=a \oplus(a \otimes b) \oplus b_{1}=a \oplus b=a+b ; \\
& a \otimes b_{1}=a \otimes b \otimes b_{1}=0 \in \mathfrak{a} \subset L,
\end{aligned}
$$

whence $\left(a, b_{1}\right) M$ by Lemma 2 . But $a b_{1}=0$, and it follows from the corollary to Lemma 2 that $\left(b_{1}, a\right) \perp$. Thus $b_{1}$ is a (left) complement within $b$ of $a$ in $a+b$. 
Theorem 7. The set a is a modular ideal in $L$ containing the set $M_{L}^{\prime}$.

Proof. It is obvious that $\mathfrak{a}$ is an ideal; that it is modular follows from the corollary to Theorem 5, equations (7) and Lemma 2. Now if $(b, c) M^{\prime}$, then by Lemma $2 b \otimes c \notin L$, whence $b c=(b \otimes c) \otimes \infty$, and $b c \in \mathfrak{a}$. Hence $M_{L}^{\prime} \subset \mathfrak{a}$.

It should be observed that both possibilities $M_{L}^{\prime}=\mathfrak{a}$ and $M_{L}^{\prime} \neq \mathfrak{a}$ can occur. In our special example where $\Lambda$ is a projective geometry and $\Lambda-L$ consists of all subelements $x$ of a hyperplane $h$ with $x$ not $\leqq \infty, M_{L}^{\prime}=\mathfrak{a}$ if there exists $k \notin L$ with $\infty<k<h$. On the other hand, if no element exists (in $\Lambda$ ) between $\infty$ and $h$, then $M_{L}^{\prime} \neq \mathfrak{a}$, for then $\infty \notin M_{L}^{\prime}$. This example shows also that $M_{L}^{\prime}$ need not be an ideal.

IllinoIs Institute of TEChNOLOGY

\title{
SUFFICIENT CONDITIONS THAT POLYNOMIALS IN SEVERAL VARIABLES BE POSITIVE
}

\author{
IRWIN E. PERLIN
}

1. Introduction. Let $T(x)$ denote a polynomial in a single real variable $x$ with real coefficients. T. Popoviciu ${ }^{1}$ established sufficient conditions that $T(x)$ be positive for all real $x$. In this paper we shall consider polynomials in several real variables with real coefficients, and establish sufficient conditions that the polynomials be positive for real values of the variables.

2. Polynomials in two real variables. In this section we shall develop sufficient conditions that a polynomial in two real variables with real coefficients be positive for all real values of the variables. Let us consider then,

$$
T(x, y)=\sum_{i=0}^{2 m} \sum_{j=0}^{2 n} b_{i j} c_{i j} x^{i} y^{j},
$$

where the $b_{i j}$ are positive constants, and the $c_{i j}$ are real numbers. Introducing parameters, we write $T(x, y)$ in the following form

Presented to the Society, April 13, 1940; received by the editor June 26, 1941.

$1 \mathrm{~T}$. Popoviciu, Sur une condition suffisante pour qu'un polynôme soit positif, Mathematica, vol. 11 (1935), pp. 247-256. 